\title{
Sugar cane manufacturing is associated with tuberculosis in an indigenous population in Brazil
}

\author{
Flávia Patussi Correia Sacchia,b, Mariana Garcia Croda a, Anderson Oliveira Estevanb, \\ Albert I. Koc,d and Julio Crodab,*
}

${ }^{a}$ University Hospital, Federal University of Grande Dourados, Brazil; ${ }^{b}$ Faculty of Health Sciences, Federal University of Grande Dourados, Brazil; ' Gonçalo Moniz Institute, Oswaldo Cruz Foundation, Brazilian Ministry of Health, Salvador, Brazil; ${ }^{d}$ Epidemiology of Microbial Disease Division, Yale School of Public Health, New Haven, USA

*Corresponding author: Present address: Faculdade de Ciências da Saúde, Universidade Federal da Grande Dourados, Rodovia Dourados - Itaúm. Km 12, Dourados, Mato Grosso do Sul 79804-970, Brasil. Tel: +55 673410 2321; Fax: +55 673410 2320; E-mail: juliocroda@ufgd.edu.br

Received 26 June 2012; revised 8 December 2012; accepted 19 December 2012

\begin{abstract}
Background: Tuberculosis (TB) remains one of the leading causes of morbidity and mortality among indigenous peoples in Brazil, and identifying the risk factors for TB in this population secondary to specific epidemiological conditions is essential for recommending interventions aimed at disease control.
\end{abstract}

Methods: This case-control study was conducted with an indigenous population between June 2009 and August 2011 in Dourados, Brazil. Tuberculosis cases reported to the national disease surveillance programme were paired with two control cases matched by age and geographic location.

Results: There were 63 cases included in this study, and the annual incidence of TB in the indigenous communities examined was 222 (95\% CI, 148-321) per 100000 inhabitants. The multivariate analysis demonstrated that the variables associated with TB infection included male gender (OR 2.6; 95\% CI 1.3-5.3), not owning a home (OR 3.4; 95\% CI 1.2-10.1), illiteracy (OR 2.4; 95\% CI 1.1-5.0), TB contact (OR 2.4; 95\% CI 1.2-4.8) and work performed in a sugar cane factory (OR 6.8; 95\% CI 1.2-36.9).

Conclusion: There is a potential relationship between exposure to sugar cane manufacturing processes and tuberculosis infection among indigenous populations.

Keywords: Risk factors, Indigenous, TB, Sugar cane, Epidemiology, Case-control study

\section{Introduction}

Despite the overall decline in the incidence of tuberculosis (TB) worldwide, ${ }^{1}$ outbreaks of TB among indigenous populations continue to challenge TB control efforts. ${ }^{2-6}$ The city of Dourados has the second largest indigenous population in Brazil. The incidence of TB for the indigenous population of Dourados is 230 per 100000 inhabitants, whereas the incidence in the nonindigenous population is 28 per 100000 inhabitants. $^{2}$ Despite the introduction and employment of the directly observed treatment short course (DOTS) in Dourados over the past 10 years and an observed reduction in the incidence of $\mathrm{TB},{ }^{2}$ new intervention measures are needed to reduce the high incidence of tuberculosis in this population.

The risk factors previously investigated in susceptible populations include alcoholism, homelessness, drug abuse, prior incarceration, HIV/AIDS infection, urban residence, positive sputum smear, male gender and prior history of TB. ${ }^{7-10}$ However, no study has identified the risk factors for indigenous populations.
The evaluation of risk factors for vulnerable populations is crucial for planning future interventions for TB control.

\section{Materials and methods}

\section{Study design}

We performed a case-control study with indigenous TB patients who resided in Dourados and had been reported to the National System on Reportable Diseases (SINAN). ${ }^{11}$ The diagnosis of pulmonary TB was based on clinical and epidemiological data, $X$-ray, thorax CT (computed tomography) and/or positive sputum smear or culture. The diagnosis of extrapulmonary TB was based on clinical and epidemiological data and complementary tests, such as cerebrospinal fluid analysis (CSF), CT and/or biopsy. The cultures were performed in solid medium, and the detection and susceptibility testing were performed using the Mycobacterial Detection System-BACTEC ${ }^{T M}$ MGIT ${ }^{\mathrm{TM}} 960$ (BD: Becton, Dickinson and Company, Franklin Lakes, NJ, USA). The 
control subjects were individuals with no previous or current TB disease (data obtained by asking the participants), and the age of each control was within 10 years of the matched diseased patient's age. The interviews were conducted between June 2009 and August 2011 and took place within one week after patients were notified that TB treatment was necessary.

\section{Study setting}

Dourados is a municipality in the state of Mato Grosso do Sul, Brazil, with a population of 189762 individuals. Specifically, the Special Indigenous Health Agency (SESAI) is responsible for health management and the TB control programme that manages the diagnosis and treatment of TB and notifies the SINAN about TB infections among the indigenous population. Dourados contains approximately 12602 indigenous people who live in peri-urban areas, and the indigenous population receives different housing, primary health care and social welfare programmes than the non-indigenous population. Overall, the indigenous population has a low education level and a high unemployment rate. The occupations available to this population are concentrated in labour-intensive activities on farms, in the charcoal industry and in the meat packing industry.

\section{Study population}

The study included indigenous TB patients who were reported to the SINAN and resided in the city of Dourados. The exclusion criteria were being a prison inmate or a non-indigenous person or having no confirmed diagnosis of TB.

For each TB patient that was included in the study, two controls were selected from the geographic region of the patient's residence; the controls resided up to $500 \mathrm{~m}$ from the patient's house. Interviews were conducted with all of the TB patients and control subjects using a standardised, pre-coded questionnaire. The questionnaire was administered by medical students who were involved in the data collection, and the process was overseen by a health worker who understood the indigenous dialect. For patients who did not understand Portuguese, the health worker translated the question and the answer provided by the participant. The same interviewer administered the questionnaire to the cases and their corresponding control subjects, and all collected data were double-checked for accuracy.

\section{Variables and study instruments}

The interviews provided data about sex, home ownership (yes or no), individual income (median $\leq$ US\$100 or >US\$100), illiteracy (i.e., having never been to school), smoking, drug use, diabetes and HIV/AIDS (self-reported), contact with a TB-positive individual (in the household or in other places), the presence of a Bacillus Calmette-Guérin (BCG) vaccine scar (determined by inspecting the arm of the participant), previous incarceration and occupation. An index of domestic over-crowding was determined by dividing the number of people who were living in the household by the number of rooms. The participant's race/skin colour was self-reported (white, black, indigenous, Asian, brown and unknown) and subsequently stratified into indigenous and non-indigenous.
The alcohol intake section of the questionnaire consisted of four questions. Alcoholism was established using the CAGE (cut down, annoyed, guilty and eye opener) questionnaire. ${ }^{12,13}$

Social class was categorised according to the classifications set forth by the Brazilian Association of Research Institutes (ABEP), which adopted the Brazilian Economic Classification Criterion (CCEB). The study population was subsequently grouped according to the number of 10 -point intervals (class D or E) that fell below the median. ${ }^{14}$

\section{Statistical analysis and sample size estimation}

All of the data were entered in duplicate into the electronic database EpiData version 3.1 (The EpiData Association, Odense, Denmark) and analysed using SAS version 9.1 (SAS Institute, Cary, NC, USA). Univariate and multivariate logistic regressions were conducted to identify the factors associated with active TB. Variables were included in the model if they achieved a significance level of $p<0.20$ in the univariate analysis. Correlated variables were tested individually, and the Wald test was used to evaluate the significance of risk factors in the final model. Statistical significance was set at $p<0.05$. The results were expressed as OR with 95\% CI. A correlation matrix was used to assess confounding variables and correlations between variables.

The sample size was calculated to reach $80 \%$ statistical power to detect an absolute difference of 10 percentage points between the cases and controls, with an $\alpha$ error of 0.05 .

\section{Ethical issues}

All of the eligible patients and the control subjects were informed about the study, and the questionnaire was administered only after receiving written confirmation of informed consent. The consent forms were read to the participants who were illiterate, and these participants provided their consent using their fingerprint. The study was conducted with the approval of the Research Ethics Committee of the Federal University of Grande Dourados (No. 002/2009) and the National Committee for Ethics in Research (No. 627/09). The potential participants who declined to participate or did not participate for other reasons were eligible for treatment (if applicable) and did not suffer in any way by not participating in the study.

\section{Results}

Between June 2009 and August 2011, 186 TB cases were reported to the SINAN. A total of 122 cases were excluded; of these, 36 were inmates, 10 did not have confirmed diagnoses of TB, and 76 were non-indigenous people, according to the selfreport of the respondents. One patient died before the interview. The study included a total of 63 patients from SINAN who selfreported that they were indigenous, living in the indigenous reservation in Dourados (Figure 1 ).

The annual incidence of TB in the indigenous communities during the study period was 222 (95\% CI 148-321) per 100000 inhabitants. Among the 63 indigenous patients, $84 \%$ (53) were diagnosed with pulmonary TB and 16\% (10) with extrapulmonary TB. Among these 10 patients, three were neurotuberculosis (based only on clinical and CSF findings without 


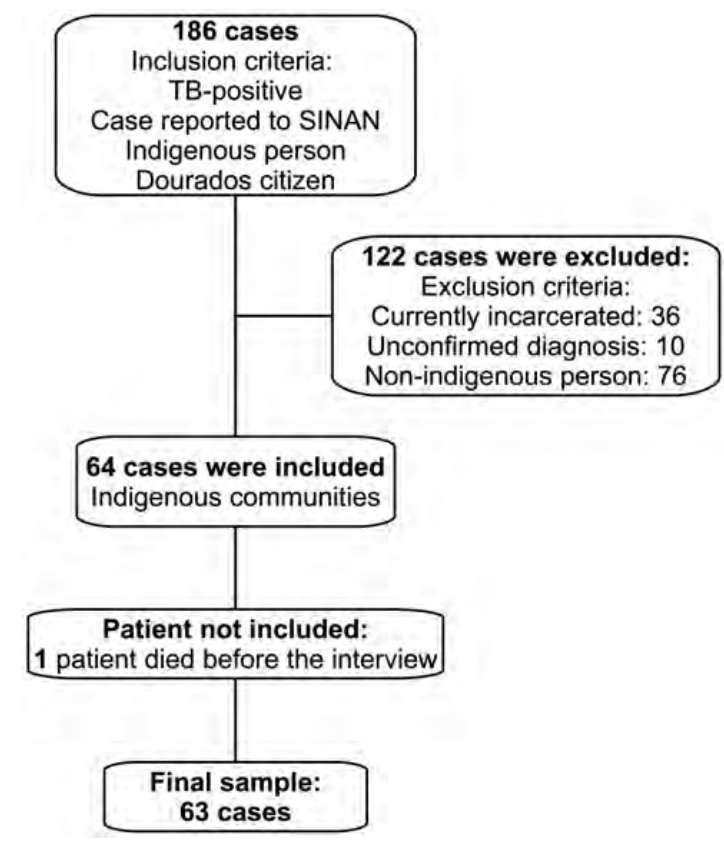

Figure 1. Flowchart illustrating the recruitment of TB cases. SINAN: National System on Reportable Diseases

definitive microbiologic confirmation), two were genitourinary TB (one culture-positive and one renal biopsy reported chronic granulomatous lymphadenitis), one was pleural TB (culturepositive and pleural biopsy reported chronic granulomatous lymphadenitis), one was lymphadenitis TB (culture-positive and lymph node biopsy reported chronic granulomatous lymphadenitis) and three were pulmonary and lymphadenitis TB (two smear-positive, three culture-positive). All 53 patients with pulmonary TB performed a sputum smear, culture, and X-ray. Of these, $77 \%$ (41) were confirmed bacteriologically (55\% [29] were smear- and culture-positive, 13\% [7] were smear-positive and culture-negative and 10\% [5] were smear-negative and culture-positive). Twenty-three per cent (12) were treated according to the changes found in the X-ray or thorax $\mathrm{CT}$. In our study, no difference was found regarding to pattern of $X$-ray and thorax CT in the indigenous population as compared with the non-indigenous population.

In smear- or culture-positive patients, TB response was evaluated monthly by smear negativity until the sixth month of treatment, and in smear-negative patients, TB response was evaluated as clinical improvement until the third month of treatment. ${ }^{15}$ No MDR strains were identified among the isolates, according to the tests performed in the MGIT 960.

Of the 63 patients included in the study, 39 (62\%) were employed, and of these, 13 patients worked in rural jobs, 10 worked in sugar cane mills, 10 worked as domestic labourers, five were bricklayers and one was a health professional.

The significant factors in the univariate analysis included male gender (OR 3.8; 95\% CI 2.0-7.1), homeowner status (OR 5.0; 95\% CI 1.9-13.1), illiteracy (OR 2.4; 95\% CI 1.3-4.7), previous contact with a TB-positive patient (OR 2.0; 95\% CI 1.1-3.7), the presence of a BCG scar (OR 0.4; $95 \%$ CI $0.2-0.9$; protective effect), prior incarceration (OR 2.9; 95\% CI 1.0-8.8) and work in a sugar cane factory (OR 10.3; 95\% CI 2.1-49.0). In the correlation matrix no variable showed an association higher than 0.4. In the multivariate analysis the final model included male gender (OR 2.6; 95\% CI 1.3-5.3), homeowner status (OR 3.4; 95\% CI 1.2-10.1), illiteracy (OR 2.4; 95\% CI 1.1-5.0), history of TB contact (OR 2.4; 95\% CI 1.2-4.8), and working in a sugar cane factory (OR 6.8; 95\% CI 1.2-36.9) (Table 1).

\section{Discussion}

Prior studies have shown that the distribution of TB is heterogeneous in developed and developing countries and that this distribution is usually influenced by demographic, socio-cultural and geographic characteristics. ${ }^{4,16}$ Tuberculosis constitutes a major health problem among indigenous populations in Brazil. The incidence of disease in these populations is high, and the rates of infection are up to ten times greater than those found in the general Brazilian population. . $^{3,4}$

The city of Dourados has recently expanded its sugar cane activity. Due to the increased demand from sugar cane mills, labour migration to the region has intensified, although many of these workers are residents of Dourados who primarily live in indigenous communities. Our results indicated that TB infection in the indigenous communities of Dourados was associated with working in sugar cane mills. Previous studies have highlighted the association between tuberculosis and occupation, as associations with TB also exist among miners, ${ }^{17}$ farmers ${ }^{18}$ and healthcare workers. ${ }^{19}$ However, the relationship between TB and employment in the sugar cane industry has not previously been reported.

This association may be explained by analysing the working conditions of this population. Although mechanised sugar cane harvesting is on the rise, much of this crop is still manually cut, which requires burning dry leaves to facilitate the harvesting and cutting of the stalks as close to the ground as possible. The indigenous population is generally involved in manual sugar cane cutting, and this type of labour is associated with low pay, low job qualifications, excessive work hours, inadequate safety gear, and an absence of sanitary and health services. ${ }^{20}$ Sugar cane workers are in frequent contact during bus rides from their homes and in the lodging facilities provided by the industry. Furthermore, the practise of burning sugar cane has been associated with respiratory disease in populations residing near sugar cane fields, ${ }^{21}$ and studies have reported an increased risk of lung cancer among sugar mill workers. ${ }^{22}$ During the burning process, combustion is incomplete, forming compounds that can irritate the respiratory system, cause inflammation and lower the immune system's defences. ${ }^{23,24}$

Previous studies have also demonstrated strong relationships between TB and the socioeconomic status of exposed populations, ${ }^{25,26}$ and our results concerning the education level of our population (as measured by literacy and homeowner status) support this observation. TB is particularly common among the poor, and lower socioeconomic status correlates with other risk factors, such as overcrowding and inadequate nutrition. ${ }^{7}$ In the indigenous population we studied, being illiterate and not owning a home were risk factors for active TB infection. These relationships are seldom discussed in relation to this population, but they are directly linked to socioeconomic status. 
Table 1. Univariate and multivariate analyses for patients with TB and control subjects from an indigenous population $(n=189)$

\begin{tabular}{|c|c|c|c|c|}
\hline Variables & Patients with TB (\%) & Controls (\%) & Crude OR (95\% CI) & Adjusted OR (95\% CI) \\
\hline \multicolumn{5}{|l|}{ Sex } \\
\hline Female & $27 / 63(42.9)$ & $93 / 126(73.8)$ & 1 & 1 \\
\hline Male & $36 / 63(57.1)$ & $33 / 126(26.2)$ & $3.8(2.0-7.1)^{\mathrm{a}, \mathrm{b}}$ & $2.6(1.3-5.3)$ \\
\hline \multicolumn{5}{|c|}{ Homeowner } \\
\hline Yes & 47/61 (77.1) & $117 / 124(94.4)$ & $1^{\mathrm{b}}$ & 1 \\
\hline No & $14 / 61(22.9)$ & $7 / 124(5.6)$ & $5.0(2.0-13.1)^{\mathrm{a}}$ & $3.4(1.2-10.1)$ \\
\hline \multicolumn{5}{|c|}{ Index of domestic over-crowding } \\
\hline Yes & $46 / 60(76.7)$ & $99 / 126(78.6)$ & $0.9(0.4-1.9)$ & NA \\
\hline No & $14 / 60(23.3)$ & $27 / 126(21.4)$ & 1 & NA \\
\hline \multicolumn{5}{|c|}{ Individual income $\leq$ US\$100 } \\
\hline Yes & $51 / 60(85.0)$ & $98 / 126(77.8)$ & $1.6(0.7-3.7)$ & NA \\
\hline No & 9/60 (15.0) & $28 / 126(22.2)$ & 1 & NA \\
\hline \multicolumn{5}{|l|}{ Illiterate } \\
\hline Yes & 47/63 (74.6) & $69 / 126(54.8)$ & $2.4(1.3-4.7)^{\mathrm{a}, \mathrm{b}}$ & $2.4(1.1-5.0)$ \\
\hline No & $16 / 63(25.4)$ & $57 / 126(45.2)$ & 1 & 1 \\
\hline \multicolumn{5}{|c|}{ Social class $\leq 10$ from the median ( $D$ or $E$ ) } \\
\hline Yes & $52 / 60(86.7)$ & $106 / 124(85.5)$ & $1.1(0.5-2.7)$ & NA \\
\hline No & $8 / 60(13.3)$ & $18 / 124(14.5)$ & 1 & NA \\
\hline \multicolumn{5}{|l|}{ Smoking } \\
\hline Yes & $8 / 63(12.7)$ & $17 / 126(13.5)$ & $0.9(0.4-2.3)$ & NA \\
\hline No & $55 / 63(87.3)$ & $109 / 126(86.5)$ & 1 & NA \\
\hline \multicolumn{5}{|l|}{ Alcoholism } \\
\hline Yes & $5 / 63(7.9)$ & 9/126 (7.1) & $1.1(0.4-3.5)$ & NA \\
\hline No & $58 / 63(92.1)$ & $117 / 126(92.9)$ & 1 & NA \\
\hline \multicolumn{5}{|l|}{ Drug use } \\
\hline Yes & $4 / 63(6.4)$ & $2 / 126(1.6)$ & $4.2(0.8-23.6)^{a}$ & NA \\
\hline No & $59 / 63(93.6)$ & $124 / 126(98.4)$ & 1 & NA \\
\hline \multicolumn{5}{|l|}{ Diabetes } \\
\hline Yes & $6 / 63(9.5)$ & $10 / 126(7.9)$ & $1.2(0.4-3.5)$ & NA \\
\hline No & $57 / 63(90.5)$ & $116 / 126(92.1)$ & 1 & NA \\
\hline \multicolumn{5}{|l|}{ HIV/AIDS } \\
\hline Yes & $1 / 63(1.59)$ & $0 / 110$ & $6.1^{c}(0.2-151.2)^{a}$ & NA \\
\hline No & $62 / 63(98.41)$ & $126 / 126$ & 1 & NA \\
\hline \multicolumn{5}{|c|}{ History of contact with TB } \\
\hline Yes & $32 / 63(50.8)$ & 43/126 (34.1) & $2.0(1.1-3.7)^{a, b}$ & $2.4(1.2-4.8)$ \\
\hline No & $31 / 63(49.2)$ & $83 / 126$ (65.9) & 1 & 1 \\
\hline \multicolumn{5}{|c|}{ BCG vaccine scar } \\
\hline Yes & $48 / 63(76.2)$ & $112 / 126(88.9)$ & $0.4(0.2-0.9)^{a, b}$ & NA \\
\hline No & $15 / 63(23.8)$ & 14/126 (11.1) & 1 & NA \\
\hline \multicolumn{5}{|l|}{ Ex-inmate } \\
\hline Yes & $8 / 63(12.7)$ & $6 / 126(4.8)$ & $2.9(1.0-8.8)^{a, b}$ & NA \\
\hline No & $55 / 63(87.3)$ & $120 / 126(95.2)$ & 1 & NA \\
\hline \multicolumn{5}{|c|}{ Sugar cane worker } \\
\hline Yes & $9 / 63(14.3)$ & 2/125 (1.6) & $10.3(2.1-49.0)^{\mathrm{a}, \mathrm{b}}$ & $6.8(1.2-36.9)$ \\
\hline No & $54 / 63(85.7)$ & $123 / 125(98.4)$ & 1 & 1 \\
\hline
\end{tabular}

NA: Not applicable.

${ }^{a}$ Variables with $p<0.2$ were included in the stepwise multiple logistic regression; ${ }^{b}$ Variables with $p<0.05$;

${ }^{\mathrm{c}}$ These logit estimators use a correction of 0.5 in every cell that contains a zero. 
Studies have shown that a history of previous contact with TB is an important factor in the epidemiology of the disease, ${ }^{25,27}$ and our results corroborate these findings. In the indigenous communities we studied, the individuals who reported previous contact with TB-infected patients had an OR for acquiring the disease that was approximately three times that of the individuals who had no history of contact. Controlling contact is an important tool for preventing illness and diagnosing early cases of active disease; it should be a priority for TB control programmes. ${ }^{28}$

Although the present study provides useful, epidemiologically relevant information that can be directly applied to various settings, it also had certain limitations. Selection bias is an important methodological limitation related to our case-control study design. Interviews with both the TB patients and the control participants were typically conducted during the day. As a result, there may have been a higher tendency to select females as controls, as females were home more often than males at the time of the interviews. Moreover, in many households, none of the residents were present at the time of the interviews, and some of these residents may have been eligible for the study. The interviewer did not return to households to ensure that there were no other eligible control subjects and instead selected control subjects from the next eligible household.

Although the findings of the present study may not be generalisable to other epidemiological situations, the identification of risk factors for TB in Dourados will likely be relevant to indigenous populations in developing countries who are subject to similar conditions. Brazilian studies have indicated that the impact of tuberculosis on indigenous peoples is substantial, and it has become a public health priority. ${ }^{29,30}$ In this context, and based on the individual risk factors of this population, numerous studies have indicated the need to implement prevention and control measures that are specifically targeted towards the indigenous population. ${ }^{3,4}$ To the best of our knowledge, this study is the first to describe the risk factors for tuberculosis in an indigenous population, and these findings may be relevant for other indigenous communities.

Authors' contributions: FPCS, MGC, and JC conceived and designed the study. FPCS, AOE and JC performed and interpreted the data analysis. FPCS, MGC, AOE and JC were responsible for writing the manuscript. JC and AIK critically revised the manuscript. All authors read and approved the final version of the paper. JC is guarantor of the paper.

Acknowledgements: We would like to thank Aline Mara da Silva Alves, Débora Rigo Guimarães de Macedo Bento, Gabriela Zampieri D’andrea, Giovana de Castro Oliveira, Gisele Douradinho Teixeira, Junio Pereira Pardins, Marcella Paranhos Rodrigues, Marcos Massaki Ota, Natália Daiane Garoni Martins, Renato Fernando Cazanti, Tiago Andrade de Oliveira e Silva, Valquiria Merjan de Figueiredo, Vanessa da Costa Maldonado, and Vinícius Nonato de Oliveira for performing the interviews of the patients and the control subjects.

Funding: This work was supported by the Foundation for the Support of Teaching, Science, and Technology Development of Mato Grosso do Sul (Fundação de Apoio ao Desenvolvimento do Ensino, Ciência e Tecnologia do Estado do Mato Grosso do Sul) [07/2009] and by the National Council for Scientific and Technological Development (Conselho Nacional de Desenvolvimento Cientifico e Tecnológico) [559094/2009-4].

Competing interests: None declared.

Ethical approval: This study was conducted with the approval of the Research Ethics Committee from the Federal University of Grande Dourados (No. 002/2009) and the National Committee for Ethics in Research (No. 627/09).

\section{References}

1 WHO. Global Tuberculosis Control. Geneva: World Health Organization; 2011.

2 Croda MG, Trajber Z, da Lima R, Croda J. Tuberculosis control in a highly endemic indigenous community in Brazil. Trans R Soc Trop Med Hyg 2011;106:223-9.

3 Basta PC, Coimbra Junior CE, Camacho LA, Santos RV. Risk of tuberculous infection in an indigenous population from Amazonia, Brazil. Int J Tuberc Lung Dis 2006;10:1354-9.

4 Basta PC, Coimbra Junior CE, Escobar AL, Santos RV. Epidemiologic aspects of tuberculosis in the Surui Indians, Brazilian Amazon. Rev Soc Bras Med Trop 2004;37:338-42.

5 Amarante JM, Costa VA. Tuberculosis in Brazilian indigenous communities at the turn of the century. Bol Pneumol Sanit 2000;8:123-8.

6 Buchillet D, Gazin P. Tuberculosis among the Amerindian population of the upper Rio Negro region. Cad Saude Publica 1998;14:181-5.

7 Nava-Aguilera E, Andersson N, Harris E et al. Risk factors associated with recent transmission of tuberculosis: systematic review and meta-analysis. Int J Tuberc Lung Dis 2009;13:17-26.

8 de la Haye B, Wild SH, Stevenson J et al. Tuberculosis and alcohol misuse in Scotland: a population-based study using enhanced surveillance data. Int J Tuberc Lung Dis 2012;16:886-90.

9 Goetsch U, Bellinger OK, Buettel KL, Gottschalk R. Tuberculosis among drug users and homeless persons: impact of voluntary X-ray investigation on active case finding. Infection 2012;40:389-95.

10 Sanchez A, Larouze B, Espinola AB et al. Screening for tuberculosis on admission to highly endemic prisons? The case of Rio de Janeiro State prisons. Int J Tuberc Lung Dis 2009;13:1247-52.

11 SINAN MS. National notifiable diseases surveillance system (Sistema de Informação de Agravos de Notificação [SINAN]). 2011.

12 Mayfield D, McLeod G, Hall P. The CAGE questionnaire: validation of a new alcoholism screening instrument. Am J Psychiatry 1974;131:1121-3.

13 Ewing JA. Detecting alcoholism: the CAGE questionnaire. JAMA 1984;252:1905- 7 .

14 ABEP. Brazilian Economic Classification Criterion [in Portuguese]. 2011. www.abep.org [accessed 01/06/2012].

15 Ministério da Saúde. Secretaria de Vigilância em Saúde. Departamento de Vigilância Epidemiológica. Guidelines for tuberculosis control in Brazil. [in Portuguese] 2011.

16 Tocque K, Regan M, Remmington Tet al. Social factors associated with increases in tuberculosis notifications. Eur Respir J 1999;13:541-5.

17 Barboza CE, Winter DH, Seiscento M et al. Tuberculosis and silicosis: epidemiology, diagnosis and chemoprophylaxis. J Bras Pneumol 2008;34:959-66.

18 Severo NPF, Leite CQF. Characterization of the population with tuberculosis in the city of Americo Brasiliense/SP, in the period 1992 to 2002. Rev Cienc Farm Bas 2005;26:83-6. 
19 Joshi R, Reingold AL, Menzies D, Pai M. Tuberculosis among health-care workers in low- and middle-income countries: a systematic review. PLoS Med 2006;3:e494.

20 Martinelli LA, Filoso S. Expansion of sugarcane ethanol production in Brazil: environmental and social challenges. Ecol Appl 2008;18:885-98.

21 Ribeiro H. Sugar cane burning in Brazil: respiratory health effects. Rev Saude Publica 2008;42:370-6.

22 Amre DK, Infante-Rivard C, Dufresne A et al. Case-control studies of lung cancer among sugar cane farmers in India. Occup Environ Med 1999; 56:548-52.

23 Arbex MA, Cançado JED, Pereira LAA et al. Biomass burning and its effects on health. J Bras Pneumol 2004;30:158-75.

24 Allen AG, Cardoso AA, Rocha GO. Influence of sugar cane burning on aerosol soluble ion composition in southeastern Brazil. Atmospheric Environment 2004;38:5025-38.

25 Menezes AMB, da Costa JD, Gonçalves $\mathrm{H}$ et al. Incidence and risk factors for tuberculosis in Pelotas, a city in the South of Brazil. Rev Bras Epidemiol 1998;1:11.
26 Dalcolmo MP, Andrade MK, Picon PD. Multiresistant tuberculosis in Brazil: history and control. Rev Saude Publica 2007;41:34-42.

27 Pakasi TA, Karyadi E, Dolmans WM et al. Malnutrition and socio-demographic factors associated with pulmonary tuberculosis in Timor and Rote Islands, Indonesia. Int J Tuberc Lung Dis 2009;13:755-9.

28 WHO. The Global Fund to Fight Aids, Tuberculosis and Malaria. Framework for Operations and Implementation Research in Health and Disease Control Programs. Geneva: World Health Organization; 2008.

29 Marques AM, da Cunha RV. Assisted treatment and tuberculosis cure and treatment dropout rates in the Guarani-Kaiwa Indian nation in the municipality of Dourados, Mato Grosso do Sul, Brazil. Cad Saude Publica 2003;19:1405-11.

30 Basta PC, Camacho LA. Tuberculin skin test to estimate the prevalence of Mycobacterium tuberculosis infection in indigenous populations in the Americas: a literature review. Cad Saude Publica 2006;22:245-54. 